This item was submitted to Loughborough's Research Repository by the author.

Items in Figshare are protected by copyright, with all rights reserved, unless otherwise indicated.

\title{
Non-linear disturbance observer-based robust control for systems with mismatched disturbances/uncertainties
}

PLEASE CITE THE PUBLISHED VERSION

http://ieeexplore.ieee.org/xpl/articleDetails.jsp?arnumber=6086648

PUBLISHER

(C) Institution of Engineering and Technology

VERSION

AM (Accepted Manuscript)

LICENCE

CC BY-NC-ND 4.0

REPOSITORY RECORD

Yang, Jun, Wen-Hua Chen, and Shihua Li. 2012. "Non-linear Disturbance Observer-based Robust Control for Systems with Mismatched Disturbances/uncertainties”. figshare. https://hdl.handle.net/2134/11123. 
This item was submitted to Loughborough's Institutional Repository (https://dspace.lboro.ac.uk/) by the author and is made available under the following Creative Commons Licence conditions.

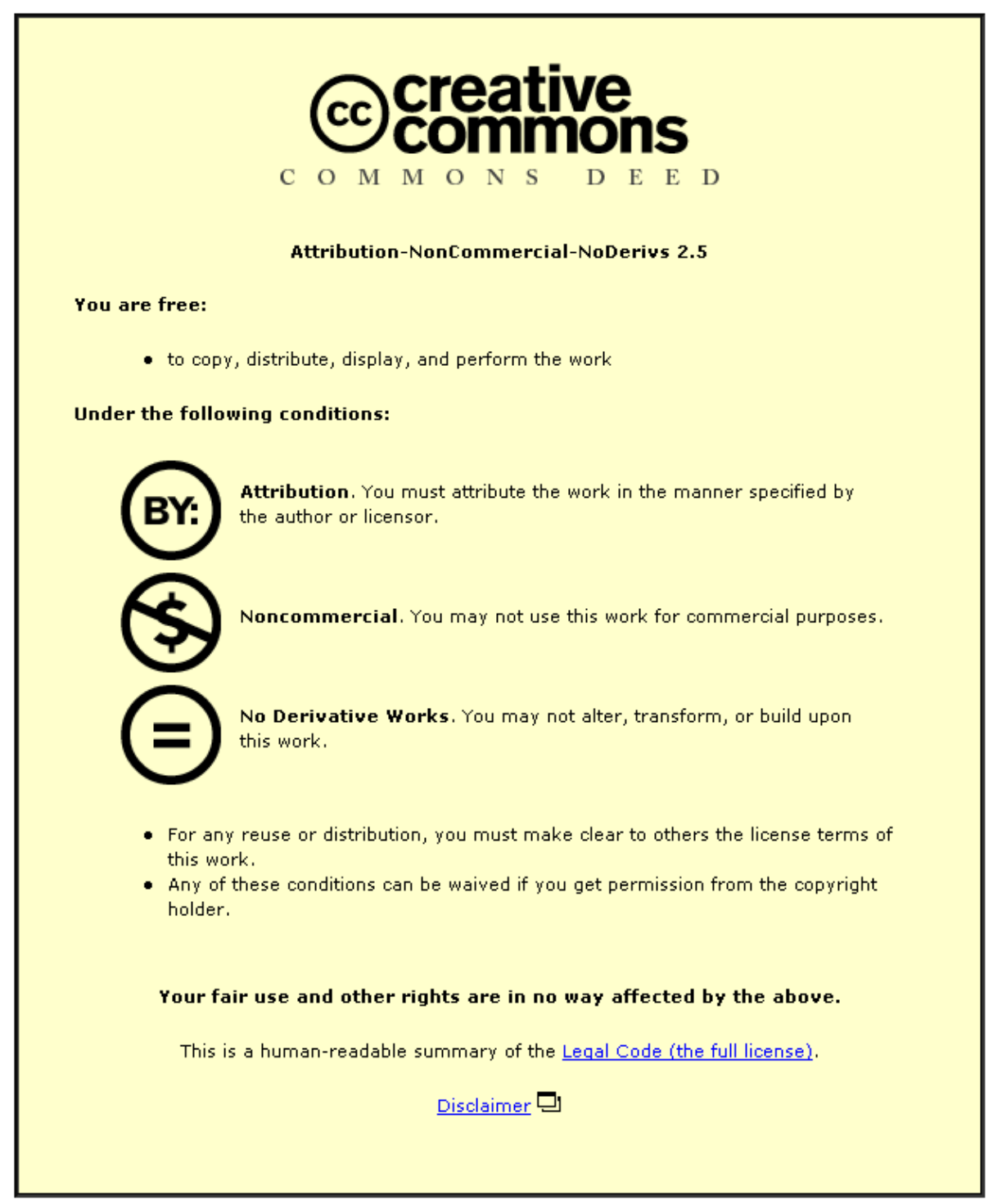

For the full text of this licence, please go to: http://creativecommons.org/licenses/by-nc-nd/2.5/ 


\title{
Nonlinear disturbance observer based robust control for systems with mismatched disturbances/uncertainties
}

\author{
Jun Yang*, Wen-Hua Chen ${ }^{\dagger}$, and Shihua $\mathrm{Li}^{*}$
}

\begin{abstract}
Robust control of nonlinear systems with disturbances and uncertainties is addressed in this paper using disturbance observer based control technique. In this framework, the "disturbance" is a generalized concept, which may include external disturbances, unmodeled dynamics, and system parameter perturbations. The existing disturbance observer based control (DOBC) methods were only applicable for the case where disturbances and uncertainties satisfy so called matching condition, i.e., they enter the system in the same channel as the control inputs. By appropriately designing a disturbance compensation gain vector in the composite control law, a nonlinear disturbance observer based robust control (NDOBRC) method is proposed in this paper to attenuate the mismatched disturbances and the influence of parameter variations from system output channels. The proposed method is applied to a nonlinear missile system in the presence of various uncertainties and external disturbances. Simulation shows that, compared with the widely used nonlinear dynamic inversion control (NDIC) and NDIC plus integral action (NDIC+I) methods, the proposed method provides much better disturbance attenuation ability and stronger robustness against various parameter variations. The proposed method significantly extends the applicability of the DOBC methods.
\end{abstract}

Keywords: Mismatched disturbances/uncertainties, nonlinear disturbance observer, disturbance compensation gain, nonlinear missile.

\section{Introduction}

Disturbances including external disturbances, unmodeled dynamics, and parameter perturbations, widely exist in aerospace engineering, such as aircrafts, missiles, and satellites, and also many other engineering systems. Generally speaking, the control performance of these systems is severely affected by disturbances and uncertainties. Disturbance attenuation is of great importance in control system design.

Disturbance observer based control (DOBC) provides a promising approach to handle system

* J. Yang and S.H. Li are with School of Automation, Southeast University, Nanjing 210096, P.R. China, emails: junyang8402@gmail.com and 1sh@seu.edu.cn

${ }^{\dagger}$ W.-H Chen is with Department of Aeronautical and Automotive Engineering, Loughborough University, LE11 3TU, UK, email: w.chen@lboro.ac.uk 
disturbances and improve robustness $[1,2]$. In this framework, a base line controller is firstly designed under the assumptions that there are no disturbances and uncertainties, and then a compensation is added to counteract the influence of disturbances and uncertainties that is estimated by a properly designed disturbance observer (DOB) [3].

Compared with other robust control schemes, DOBC approach has two distinct features. One feature is that disturbance observer based compensation can be considered as a "patch" for existing controllers that may provide good stability and tracking performance but have unsatisfactory disturbance attenuation and robustness against uncertainties. The benefits of this is that there is no change to the base line controller which may have been widely used and developed for many years such as classical flight control systems. After the base line controller is designed using the existing procedures, the disturbance observer based compensation is added to improve the robustness and disturbance attenuation. Instead of employing a complete new and different control strategy which demands a new verification and certification process, the verification of DOBC can be developed based on the existing verification process to ensure safety and reliability. The second feature is that DOBC is not a worst case based design. Most of the existing robust control methods are worst case based design, and have been criticized as being "over conservative". Promising robustness is achieved with the price of degraded nominal performance. In DOBC approach, the nominal performance of the base line controller is recovered in the absence of disturbances or uncertainties. Although in the worst cases for described uncertainties, DOBC's performance may be poorer than that of other robust controllers, the overall better performance may be achieved as a dynamic system more likely operates around its nominal condition.

Disturbance observer technique was firstly presented by Ohishi et al. [4] for a motion servo system in the late of 1980s. During the past two decades, DOBC schemes for linear and nonlinear systems have been successfully developed and applied in various engineering systems, such as servo control system [2], robotic system [5], hard disk drive system [6], position system [7], grinding system $[8,9]$, etc.

Note that the above mentioned DOBC schemes are generally designed according to linear control theory, even if the actual controlled plant is nonlinear [10]. In the presence of strong nonlinearities, the validity of using linear analysis and synthesis technique may be doubtful $[11,12,13]$. To this end, research of DOBC using nonlinear system theory has drawn much attention in the past decade. Recent developments of nonlinear disturbance observer based control (NDOBC) can be seen in $[3,11,12,13,14,15]$. In [14], a sliding mode based nonlinear disturbance observer (NDOB) was proposed for motor control. Using Lyapunov stability theory, Chen et al. [11] developed a nonlinear disturbance observer for a single-input-single-output (SISO) nonlinear system subject to unknown constant disturbance and applied it to a two-link manipulator. Rigorous exponential stability analysis of NDOBC was established based on Lyapunov stability theory in [3]. Guo and Chen [12] proposed a DOBC method for a class of multi-input-multi-output (MIMO) nonlinear systems 
with well-defined nonlinearity. Composite methods which combine DOBC with terminal sliding model control or $H_{\infty}$ control were proposed to solve the problem caused by disturbance model perturbations in Ref. [12, 13, 15].

In the previous literature, the DOBC methods were only applicable to disturbances satisfying matching condition [16]. Here matching condition means that the disturbances appear in the same channels as the control inputs. For mismatched disturbances, i.e., the disturbances or the influence of uncertainties enter the system in different channels from the control inputs, the existing DOBC methods are not applicable. It should be pointed out that mismatched disturbances are more practical and widely exist in engineering systems. Taking aircraft as an example, the lumped disturbance torques caused by unmodeled dynamics, external winds, and parameter perturbations may influence the states directly, rather than through the input channels.

The problem of mismatched disturbances have been concerned in $[13,15]$ where the matched disturbances were canceled by DOBC while the mismatched disturbances were attenuated by $H_{\infty}$ control [15] or variable structure control [13]. It was also reported that some constrains with the mismatched disturbances (such as with bounded $H_{2}$ norm) were required in $[13,15]$. In the presence of mismatched disturbances/uncertainties, a widely used practical method is to add an integral action in the feedback control law to remove offset of the closed-loop system [17], which will be compared with the proposed method in this paper.

In this paper, a nonlinear disturbance observer based robust control (NDOBRC) method is proposed to solve the disturbance attenuation problem of nonlinear systems subject to mismatched disturbances/uncertainties. In the presence of mismatched disturbances and uncertainties, it is unlikely to achieve asymptotic stability as the disturbance and the control inputs do not appear in the same channels and the influence of the disturbances can not be completely canceled. Instead, the design objective is to remove the influence of disturbances and uncertainties from the output. The key issue here is how to design a disturbance compensation gain vector to assure that the mismatched disturbances can be completely eliminated from the output channels. With the proposed NDOBRC method, the prominent "patch" feature retains, the robustness and disturbance attenuation against a much wider range of uncertainties and disturbances are significantly improved without sacrificing the nominal performance of the original nonlinear control strategy.

To demonstrate the feasibility and effectiveness of the proposed method, control design of a missile system using NDOBRC is investigated in this paper. The missile system considered is subject to mismatched disturbances that include not only external disturbances, but also model uncertainties. The rest of the paper are organized as follows. In Section 2, a preliminary regarding nonlinear disturbance observers is presented for the convenience of the readers. The problem formulation is then described. Design and analysis of the proposed NDOBRC is presented in Section 3. In Section 4, the proposed NDOBRC is applied to a nonlinear missile system. Simulation studies and results are presented in Section 5. The conclusions are finally given in Section 6. 


\section{Preliminaries and problem formulation}

\subsection{Nonlinear disturbance observer}

A general single-input-single-output (SISO) affine nonlinear systems with lumped disturbances is represented as

$$
\left\{\begin{array}{l}
\dot{\boldsymbol{x}}=\boldsymbol{f}(\boldsymbol{x})+\boldsymbol{g}_{1}(\boldsymbol{x}) u+\boldsymbol{g}_{2}(\boldsymbol{x}) \boldsymbol{d} \\
y=h(\boldsymbol{x})
\end{array}\right.
$$

where $\boldsymbol{x} \in \boldsymbol{R}^{n}, \boldsymbol{d} \in \boldsymbol{R}^{n}, u \in R$ and $y \in R$ are the state vector, lumped disturbance vector, input and output variables. It is assumed that $\boldsymbol{f}(\boldsymbol{x}), \boldsymbol{g}_{1}(\boldsymbol{x}), \boldsymbol{g}_{2}(\boldsymbol{x})$ and $h(\boldsymbol{x})$ are smooth functions in terms of state $\boldsymbol{x}$.

For system (1), the following nonlinear disturbance observer (NDOB) is proposed in $[1,3,18]$ to estimate the unknown disturbances $\boldsymbol{d}$, given by

$$
\left\{\begin{array}{l}
\hat{\boldsymbol{d}}=\boldsymbol{z}+\boldsymbol{p}(\boldsymbol{x}) \\
\dot{\boldsymbol{z}}=-\boldsymbol{l}(\boldsymbol{x}) \boldsymbol{g}_{2}(\boldsymbol{x}) \boldsymbol{z}-\boldsymbol{l}(\boldsymbol{x})\left[\boldsymbol{g}_{2}(\boldsymbol{x}) \boldsymbol{p}(\boldsymbol{x})+\boldsymbol{f}(\boldsymbol{x})+\boldsymbol{g}_{1}(\boldsymbol{x}) u\right]
\end{array}\right.
$$

where $\hat{\boldsymbol{d}}$ and $z$ are the estimates of the unknown disturbances and the internal states of the nonlinear observer, respectively, and $\boldsymbol{p}(\boldsymbol{x})$ is a nonlinear vector-valued function to be designed. The nonlinear observer gain $\boldsymbol{l}(\boldsymbol{x})$ is defined as

$$
\boldsymbol{l}(\boldsymbol{x})=\frac{\partial \boldsymbol{p}(\boldsymbol{x})}{\partial \boldsymbol{x}}
$$

Assumption 1: The lumped disturbance $\boldsymbol{d}$ is slowly time-varying, i.e., $\dot{\boldsymbol{d}} \approx 0$.

It can be proved that, under the assumption that the disturbances are slowly time-varying, $\hat{\boldsymbol{d}}$ approaches $\boldsymbol{d}$ asymptotically if $\boldsymbol{p}(\boldsymbol{x})$ is chosen such that

$$
\dot{e}(t)+\frac{\partial \boldsymbol{p}(\boldsymbol{x})}{\partial \boldsymbol{x}} \boldsymbol{g}_{2}(\boldsymbol{x}) \boldsymbol{e}(t)=0
$$

is globally stable for all $\boldsymbol{x} \in \boldsymbol{R}^{n}$, where the estimation error is defined as

$$
e=d-\hat{d}
$$

Clearly, any nonlinear vector-valued function $\boldsymbol{l}(\boldsymbol{x})$ which makes Eq. (4) asymptotically stable can be chosen to guarantee the asymptotic convergence of the estimation error.

Remark 1: The rigorous asymptotic convergence of NDOB has been established under the condition that the disturbances vary slowly relative to the observer dynamics (i.e., Assumption 1). It has been also reported that the observer (2) can track some fast time-varying disturbances with bounded error as long as the derivative of the disturbances is bounded [11].

Remark 2: In the presence of uncertainties, the lumped disturbances would be a function of the states, which can be reasonably estimated if the disturbance observer dynamics is faster than the closed-loop dynamics. The same argument for the state observer based control methods is applicable. 


\subsection{Problem formulation}

In the previous literature, the DOBC methods only deal with the case of matched disturbances, i.e., the lumped disturbances $\boldsymbol{d}$ enter the system with the same channels as the control inputs. Precisely speaking, the matched disturbances means that the following two conditions are satisfied: (i) the control inputs $u$ and the lumped disturbances $\boldsymbol{d}$ have the same dimension, and (ii) $\boldsymbol{g}_{1}(\boldsymbol{x})=\boldsymbol{g}_{2}(\boldsymbol{x})$ in Eq. (1). These conditions have restricted the application of DOBC strategies to more general controlled plants.

Remark 3: Note that the NDOB (2) is applicable for the case of mismatched disturbances. However, the estimates of NDOB can not be used to compensate the disturbances directly because the disturbances are not in the same channels with the control inputs.

It should be pointed out that in general, the influence of the mismatched disturbances can not be removed from state variables. In this paper, based on the disturbance estimate of NDOB (2), the composite control law as $u=\alpha(\boldsymbol{x})+\beta(\boldsymbol{x}) \hat{d}$ is designed to remove the influence of the lumped disturbance from the output channel by appropriately designing the compensation gain $\beta(\boldsymbol{x})$. This will substantially extends the application fields of the DOBC strategy.

A general design procedure of nonlinear disturbance observer based robust control (NDOBRC) for system (1) subject to mismatched disturbances is proposed as follows:

(i) Design a base line nonlinear feedback controller to achieve stability and performance specifications without taking into account disturbances/uncertainties.

(ii) Lump the external disturbances and the influences of the uncertainties, and then design a nonlinear disturbance observer to estimate the lumped disturbances.

(iii) Design a disturbance compensation gain vector to achieve desired performance specification in the presence of external disturbances and uncertainties.

(iv) Construct the composite NDOBRC law by integrating the nonlinear feedback controller and the disturbance observer based compensation part.

\section{NDOBRC for nonlinear systems with mismatched disturbances}

\subsection{Composite control law}

For the nonlinear systems (1) with mismatched lumped disturbances, the composite control law of NDOBRC is designed as

$$
u=\alpha(\boldsymbol{x})+\boldsymbol{\beta}(\boldsymbol{x}) \hat{\boldsymbol{d}},
$$

where $\alpha(\boldsymbol{x})$ is the feedback control law without considering the disturbances, $\boldsymbol{\beta}(\boldsymbol{x})$ is the disturbance compensation gain vector to be designed, and $\hat{d}$ is the disturbance estimate based on the NDOB (2).

Obviously, the composite control law (6) consists of two parts: one is the nonlinear feedback control part $\alpha(\boldsymbol{x})$, and the other is the disturbance compensation term $\boldsymbol{\beta}(\boldsymbol{x}) \hat{\boldsymbol{d}}$ based on the NDOB. 
Note that in the composite control law (6), the disturbance compensation term $\boldsymbol{\beta}(\boldsymbol{x}) \hat{\boldsymbol{d}}$ is just designed for disturbances, i.e., the NDOB works if and only if disturbances exist. Thus it just works like a "patch" for the existing controller to improve its disturbance attenuation and robustness against uncertainties. In the absence of disturbances and uncertainties, the nominal performance of the existing controller recovers.

\subsection{Stability analysis}

To establish the stability of the closed-loop system, the following preliminary result is required.

Lemma 1: Consider a nonlinear system $\dot{\boldsymbol{x}}=\boldsymbol{H}(\boldsymbol{x}, \boldsymbol{d})$ with state $\boldsymbol{x} \in \boldsymbol{R}^{n}$, input $\boldsymbol{d} \in \boldsymbol{R}^{m}$, in which $\boldsymbol{H}(\mathbf{0}, \mathbf{0})=\mathbf{0}$. Suppose the equilibrium $\boldsymbol{x}=\mathbf{0}$ of $\dot{\boldsymbol{x}}=\boldsymbol{H}(\boldsymbol{x}, \mathbf{0})$ is globally asymptotically stable. Then there exists an $m \times m$ matrix $\boldsymbol{M}(\boldsymbol{x})$ of smooth functions of $\boldsymbol{x}$, which is defined for all $\boldsymbol{x} \in \boldsymbol{R}^{n}$ and is nonsingular for all $\boldsymbol{x}$, such that $\dot{\boldsymbol{x}}=\boldsymbol{H}(\boldsymbol{x}, \boldsymbol{M}(\boldsymbol{x}) \boldsymbol{d})$ is input-to-state stable (ISS) [19].

Consider the lumped disturbances $\boldsymbol{d}$ as the inputs of the closed-loop system, also the system state $\boldsymbol{x}$ and observer state $\boldsymbol{e}$ as of the state of the closed-loop system. Let

$$
\begin{gathered}
\overline{\boldsymbol{x}}=\left(\begin{array}{c}
\boldsymbol{x} \\
\boldsymbol{e}
\end{array}\right), \\
\boldsymbol{F}(\overline{\boldsymbol{x}})=\left(\begin{array}{c}
\boldsymbol{f}(\boldsymbol{x})+\boldsymbol{g}_{1}(\boldsymbol{x}) \boldsymbol{\alpha}(\boldsymbol{x})-\boldsymbol{g}_{1}(\boldsymbol{x}) \boldsymbol{\beta}(\boldsymbol{x}) \boldsymbol{e} \\
-\boldsymbol{l}(\boldsymbol{x}) \boldsymbol{g}_{2}(\boldsymbol{x}) \boldsymbol{e}
\end{array}\right) .
\end{gathered}
$$

The input-to-state stability of the closed-loop system is established by the following theorem.

Theorem 1: The closed-loop system consists of nonlinear system (1), composite control law (6) and nonlinear disturbance observer (2) is ISS if the following conditions are satisfied:

(i). the nonlinear system (1) under the original designed controller $u=\alpha(\boldsymbol{x})$ is globally asymptotically stable in the absence of disturbances,

(ii). the vector-valued function $\boldsymbol{p}(\boldsymbol{x})$ is chosen such that the observer error system (4) is globally asymptotically stable,

(iii). there exist a disturbance compensation gain $\boldsymbol{\beta}(\boldsymbol{x})$ such that

$$
\boldsymbol{g}_{2}(\boldsymbol{x})+\boldsymbol{g}_{1}(\boldsymbol{x}) \boldsymbol{\beta}(\boldsymbol{x})=\boldsymbol{G}(\overline{\boldsymbol{x}}) \boldsymbol{M}(\overline{\boldsymbol{x}})
$$

holds, where $\boldsymbol{G}(\overline{\boldsymbol{x}})$ is an arbitrary matrix and $\boldsymbol{M}(\overline{\boldsymbol{x}})$ is a matrix such that

$$
\dot{\overline{\boldsymbol{x}}}=\boldsymbol{F}(\overline{\boldsymbol{x}})+\left(\begin{array}{c}
\boldsymbol{G}(\overline{\boldsymbol{x}}) \boldsymbol{M}(\overline{\boldsymbol{x}}) \\
\mathbf{0}
\end{array}\right) \boldsymbol{d}
$$

is ISS. 
Proof: Combining system (1), composite control law (6), disturbance estimation error function (4) and (5) together, the closed-loop system is obtained

$$
\left\{\begin{aligned}
\dot{\boldsymbol{x}} & =\left[\boldsymbol{f}(\boldsymbol{x})+\boldsymbol{g}_{1}(\boldsymbol{x}) \alpha(\boldsymbol{x})\right]-\boldsymbol{g}_{1}(\boldsymbol{x}) \boldsymbol{\beta}(\boldsymbol{x}) \boldsymbol{e}+\left[\boldsymbol{g}_{2}(\boldsymbol{x})+\boldsymbol{g}_{1}(\boldsymbol{x}) \boldsymbol{\beta}(\boldsymbol{x})\right] \boldsymbol{d}, \\
\dot{\boldsymbol{e}} & =-\frac{\partial \boldsymbol{p}(\boldsymbol{x})}{\partial \boldsymbol{x}} \boldsymbol{g}_{2}(\boldsymbol{x}) \boldsymbol{e}
\end{aligned}\right.
$$

Combining Eqs. (7), (8) with (11), the closed-loop system is given as

$$
\dot{\overline{\boldsymbol{x}}}=\boldsymbol{F}(\overline{\boldsymbol{x}})+\left(\begin{array}{c}
\boldsymbol{g}_{2}(\boldsymbol{x})+\boldsymbol{g}_{1}(\boldsymbol{x}) \boldsymbol{\beta}(\boldsymbol{x}) \\
\mathbf{0}
\end{array}\right) \boldsymbol{d} .
$$

With the conditions given in (i) and (ii), it can be shown that $\dot{\overline{\boldsymbol{x}}}=\boldsymbol{F}(\overline{\boldsymbol{x}})$ is asymptotically stable in the absence of disturbances. This means that there exists a $C^{1}$ positive definite and proper function $\boldsymbol{V}(\overline{\boldsymbol{x}})$ and a class $\mathcal{K}_{\infty}$ function $\gamma(\cdot)$ such that

$$
\forall \boldsymbol{x} \in \boldsymbol{R}^{n} / \mathbf{0} \Rightarrow \frac{\partial \boldsymbol{V}}{\partial \overline{\boldsymbol{x}}} \boldsymbol{F}(\overline{\boldsymbol{x}})<-\gamma(\|\boldsymbol{x}\|),
$$

where $\|\cdot\|$ denotes Euclidean norm of a vector, definitions of class $\mathcal{K}$ and $\mathcal{K}_{\infty}$ functions can be found in $[19]($ pp. 1).

Considering the following system

$$
\dot{\bar{x}}=\boldsymbol{H}(\overline{\boldsymbol{x}}, \boldsymbol{d})=\boldsymbol{F}(\overline{\boldsymbol{x}})+\left(\begin{array}{c}
\boldsymbol{G}(\overline{\boldsymbol{x}}) \\
0
\end{array}\right) \boldsymbol{d} .
$$

Combining (13) with (14) gives

$$
\forall \boldsymbol{x} \in \boldsymbol{R}^{n} / \mathbf{0} \Rightarrow \frac{\partial \boldsymbol{V}}{\partial \overline{\boldsymbol{x}}} \boldsymbol{H}(\overline{\boldsymbol{x}}, \mathbf{0})<-\gamma(\|\boldsymbol{x}\|) .
$$

Similar with the proof of Lemma 1 in [19](pp. 27-28), it can be shown that there exists a $n \times n$ matrix $\boldsymbol{M}(\boldsymbol{x})$ which is defined for all $\boldsymbol{x} \in \boldsymbol{R}^{n}$ and is nonsingular for all $\boldsymbol{x}$, such that for some class $\mathcal{K}$ function $\chi(\cdot)$

$$
\forall\|\boldsymbol{x}\| \geq \chi(\|\boldsymbol{d}\|) \Rightarrow \frac{\partial \boldsymbol{V}}{\partial \overline{\boldsymbol{x}}} \boldsymbol{H}(\overline{\boldsymbol{x}}, \boldsymbol{M}(\overline{\boldsymbol{x}}) \boldsymbol{d})<-\frac{1}{2} \gamma(\|\boldsymbol{x}\|)
$$

Consider the condition given in (iii) and (16), it can be concluded that the closed-loop system (12) is ISS.

\subsection{Design of the disturbance compensation gain}

The nonlinear systems (1) and the composite control law (6) can be rewritten and expressed as

$$
\left\{\begin{array}{l}
\dot{\boldsymbol{x}}=\overline{\boldsymbol{f}}(\boldsymbol{x}) \boldsymbol{x}+\boldsymbol{g}_{1}(\boldsymbol{x}) u+\boldsymbol{g}_{2}(\boldsymbol{x}) \boldsymbol{d} \\
y=\overline{\boldsymbol{h}}(\boldsymbol{x}) \boldsymbol{x}
\end{array}\right.
$$

and

$$
u=\overline{\boldsymbol{\alpha}}(\boldsymbol{x}) \boldsymbol{x}+\boldsymbol{\beta}(\boldsymbol{x}) \hat{\boldsymbol{d}}
$$

where $\overline{\boldsymbol{f}}(\boldsymbol{x})=\boldsymbol{f}(\boldsymbol{x}) / \boldsymbol{x}, \overline{\boldsymbol{h}}(\boldsymbol{x})=\boldsymbol{h}(\boldsymbol{x}) / \boldsymbol{x}$, and $\overline{\boldsymbol{\alpha}}(\boldsymbol{x})=\alpha(\boldsymbol{x}) / \boldsymbol{x}$. 
Theorem 2: Suppose Assumption 1 is satisfied. Consider nonlinear system (1) under the composite control law (6) consisting of the nonlinear feedback control law $\alpha(\boldsymbol{x})$ and the disturbance compensation term $\boldsymbol{\beta}(\boldsymbol{x}) \hat{\boldsymbol{d}}$ based on the estimates of the NDOB (2). The influence of the lumped disturbances can be eliminated from the output channel in steady-state if the nonlinear disturbance compensation gain $\boldsymbol{\beta}(\boldsymbol{x})$ is selected such that

(i). the closed-loop system (11) is ISS.

(ii). the following condition holds

$$
\begin{aligned}
\boldsymbol{\beta}(\boldsymbol{x})= & -\left\{\overline{\boldsymbol{h}}(\boldsymbol{x})\left[\overline{\boldsymbol{f}}(\boldsymbol{x})+\boldsymbol{g}_{1}(\boldsymbol{x}) \overline{\boldsymbol{\alpha}}(\boldsymbol{x})\right]^{-1} \boldsymbol{g}_{1}(\boldsymbol{x})\right\}^{-1} \\
& \times \overline{\boldsymbol{h}}(\boldsymbol{x})\left[\overline{\boldsymbol{f}}(\boldsymbol{x})+\boldsymbol{g}_{1}(\boldsymbol{x}) \overline{\boldsymbol{\alpha}}(\boldsymbol{x})\right]^{-1} \boldsymbol{g}_{2}(\boldsymbol{x}) .
\end{aligned}
$$

Proof: Considering the closed-loop system (11) with Eqs. (17) and (18), the states can be expressed as

$$
\boldsymbol{x}=\left[\overline{\boldsymbol{f}}(\boldsymbol{x})+\boldsymbol{g}_{1}(\boldsymbol{x}) \overline{\boldsymbol{\alpha}}(\boldsymbol{x})\right]^{-1}\left\{\dot{\boldsymbol{x}}-\boldsymbol{g}_{1}(\boldsymbol{x}) \boldsymbol{\beta}(\boldsymbol{x}) \boldsymbol{e}-\left[\boldsymbol{g}_{1}(\boldsymbol{x}) \boldsymbol{\beta}(\boldsymbol{x})+\boldsymbol{g}_{2}(\boldsymbol{x})\right] \boldsymbol{d}\right\}
$$

Combining Eqs. (19), (20) with the output equation in (17) yields

$$
y=\overline{\boldsymbol{h}}(\boldsymbol{x})\left[\overline{\boldsymbol{f}}(\boldsymbol{x})+\boldsymbol{g}_{1}(\boldsymbol{x}) \overline{\boldsymbol{\alpha}}(\boldsymbol{x})\right]^{-1} \dot{\boldsymbol{x}}+\overline{\boldsymbol{h}}(\boldsymbol{x})\left[\overline{\boldsymbol{f}}(\boldsymbol{x})+\boldsymbol{g}_{1}(\boldsymbol{x}) \overline{\boldsymbol{\alpha}}(\boldsymbol{x})\right]^{-1} \boldsymbol{g}_{2}(\boldsymbol{x}) \boldsymbol{e}
$$

Since the closed-loop system is stable, the following two conditions are satisfied, i.e., $\lim _{t \rightarrow \infty} \dot{\boldsymbol{x}}(t) \rightarrow 0$ and $\lim _{t \rightarrow \infty} \boldsymbol{e}(t) \rightarrow 0$. The later follows from the properly designed disturbance observers. It can be shown that the disturbances can be finally attenuated from the output in steady-state as $y=0$.

Remark 4: Let $\boldsymbol{g}_{2}(\boldsymbol{x})=\left(\boldsymbol{g}_{21}(\boldsymbol{x}), \boldsymbol{g}_{22}(\boldsymbol{x}), \cdots, \boldsymbol{g}_{2 n}(\boldsymbol{x})\right)$, a more explicit expression of the disturbance compensation gain can be given as

$$
\boldsymbol{\beta}(\boldsymbol{x})=\left(\beta_{1}(\boldsymbol{x}), \beta_{2}(\boldsymbol{x}), \cdots, \beta_{n}(\boldsymbol{x})\right),
$$

where

$$
\beta_{i}(\boldsymbol{x})=-\frac{\operatorname{det}\left(\begin{array}{cc}
\overline{\boldsymbol{f}}(\boldsymbol{x})+\boldsymbol{g}_{1}(\boldsymbol{x}) \overline{\boldsymbol{\alpha}}(\boldsymbol{x}) & \boldsymbol{g}_{2 i}(\boldsymbol{x}) \\
-\overline{\boldsymbol{h}}(\boldsymbol{x}) & 0
\end{array}\right)}{\operatorname{det}\left(\begin{array}{cc}
\overline{\boldsymbol{f}}(\boldsymbol{x})+\boldsymbol{g}_{1}(\boldsymbol{x}) \overline{\boldsymbol{\alpha}}(\boldsymbol{x}) & \boldsymbol{g}_{1}(\boldsymbol{x}) \\
-\overline{\boldsymbol{h}}(\boldsymbol{x}) & 0
\end{array}\right)}
$$

In fact,

$$
\begin{aligned}
\overline{\boldsymbol{h}}(\boldsymbol{x})\left[\overline{\boldsymbol{f}}(\boldsymbol{x})+\boldsymbol{g}_{1}(\boldsymbol{x}) \overline{\boldsymbol{\alpha}}(\boldsymbol{x})\right]^{-1} \boldsymbol{g}_{1}(\boldsymbol{x}) & =\frac{\overline{\boldsymbol{h}}(\boldsymbol{x}) \operatorname{adj}\left(\overline{\boldsymbol{f}}(\boldsymbol{x})+\boldsymbol{g}_{1}(\boldsymbol{x}) \overline{\boldsymbol{\alpha}}(\boldsymbol{x})\right) \boldsymbol{g}_{1}(\boldsymbol{x})}{\operatorname{det}\left(\overline{\boldsymbol{f}}(\boldsymbol{x})+\boldsymbol{g}_{1}(\boldsymbol{x}) \overline{\boldsymbol{\alpha}}(\boldsymbol{x})\right)} \\
& =\frac{\operatorname{det}\left(\begin{array}{cc}
\overline{\boldsymbol{f}}(\boldsymbol{x})+\boldsymbol{g}_{1}(\boldsymbol{x}) \overline{\boldsymbol{\alpha}}(\boldsymbol{x}) & \boldsymbol{g}_{1}(\boldsymbol{x}) \\
-\overline{\boldsymbol{h}}(\boldsymbol{x}) & 0
\end{array}\right)}{\operatorname{det}\left(\overline{\boldsymbol{f}}(\boldsymbol{x})+\boldsymbol{g}_{1}(\boldsymbol{x}) \overline{\boldsymbol{\alpha}}(\boldsymbol{x})\right)}
\end{aligned}
$$

similarly, for all $i=1,2, \cdots, n$,

$$
\overline{\boldsymbol{h}}(\boldsymbol{x})\left[\overline{\boldsymbol{f}}(\boldsymbol{x})+\boldsymbol{g}_{1}(\boldsymbol{x}) \overline{\boldsymbol{\alpha}}(\boldsymbol{x})\right]^{-1} \boldsymbol{g}_{2 i}(\boldsymbol{x})=\frac{\operatorname{det}\left(\begin{array}{cc}
\overline{\boldsymbol{f}}(\boldsymbol{x})+\boldsymbol{g}_{1}(\boldsymbol{x}) \overline{\boldsymbol{\alpha}}(\boldsymbol{x}) & \boldsymbol{g}_{2 i}(\boldsymbol{x}) \\
-\overline{\boldsymbol{h}}(\boldsymbol{x}) & 0
\end{array}\right)}{\operatorname{det}\left(\overline{\boldsymbol{f}}(\boldsymbol{x})+\boldsymbol{g}_{1}(\boldsymbol{x}) \overline{\boldsymbol{\alpha}}(\boldsymbol{x})\right)}
$$


Combining Eqs. (19), (23) and (24), yields the result of Remark 4.

Remark 5: Note that the nonlinear disturbance compensation gain vector $\boldsymbol{\beta}(\boldsymbol{x})$ in (19) is a general case and suitable for both matched and mismatched disturbances. In the matched case, i.e., $\boldsymbol{g}_{1}(\boldsymbol{x})=\boldsymbol{g}_{2}(\boldsymbol{x})$, it can be obtained from (19) that the nonlinear disturbance compensation gain vector reduces to $\boldsymbol{\beta}(\boldsymbol{x})=-1$ which is widely used in the previous DOBC designs $[1,12,13,15]$.

\section{Control design of a nonlinear missile}

\subsection{Longitudinal dynamics of a missile system}

The model of the longitudinal dynamics of a missile under consideration is taken from Refs. [1, 20], described by

$$
\begin{gathered}
\dot{\alpha}=f_{1}(\alpha)+q+b_{1}(\alpha) \delta+d_{1}, \\
\dot{q}=f_{2}(\alpha)+b_{2} \delta+d_{2},
\end{gathered}
$$

where $\alpha$ is the angle of attack (degrees), $q$ is the pitch rate (degrees per second), and $\delta$ is the tail fin deflection (degrees). The disturbances $d_{1}$ and $d_{2}$ denote the lumped disturbance torques which may be caused by unmodeled dynamics, external wind, and variation of aerodynamic coefficients, etc. The nonlinear functions $f_{1}(\alpha), f_{2}(\alpha), b_{1}(\alpha)$, and $b_{2}$ are determined by aerodynamic coefficients.

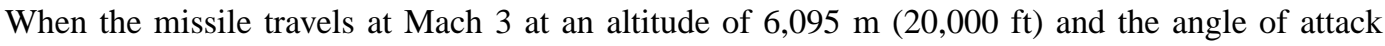
$|\alpha| \leq 20 \mathrm{deg}$, they are given by

$$
\begin{gathered}
f_{1}(\alpha)=\frac{180 g Q S}{\pi W V} \cos \left(\frac{\pi \alpha}{180}\right)\left(1.03 \times 10^{-4} \alpha^{3}-9.45 \times 10^{-3} \alpha|\alpha|-1.7 \times 10^{-1} \alpha\right), \\
f_{2}(\alpha)=\frac{180 Q S d}{\pi I_{y y}}\left(2.15 \times 10^{-4} \alpha^{3}-1.95 \times 10^{-2} \alpha|\alpha|+5.1 \times 10^{-2} \alpha\right), \\
b_{1}(\alpha)=-3.4 \times 10^{-2} \frac{180 g Q S}{\pi W V} \cos \left(\frac{\pi \alpha}{180}\right), \\
b_{2}=-0.206 \frac{180 Q S d}{\pi I_{y y}} .
\end{gathered}
$$

The tail fin actuator dynamics are approximated by a first-order lag process, i.e.,

$$
\dot{\delta}=\left(1 / t_{1}\right)(-\delta+u)+d_{3}
$$

where $u$ the commanded fin defection (degrees), $d_{3}$ the disturbance which may influence the actuator dynamics (e.g. frictions) and $t_{1}$ the time constant (seconds). The physical meaning and values of the parameters in Eqs. (27)-(31) for the missile under consideration are listed in Table 1.

\subsection{Nonlinear dynamic inversion control}

In the absence of disturbances $d_{1}, d_{2}$ and $d_{3}$, an autopilot for the missile to track an angle-of-attack reference $\omega(t)$ may be designed using the nonlinear dynamic inversion control (NDIC) [21]. The output is chosen as

$$
y=\alpha+k_{q} q
$$


Table 1: Parameters in longitudinal dynamics of the missile

\begin{tabular}{|l|c|c|}
\hline Parameter & Symbol & Value \\
\hline Weight & $W$ & $4,410 \mathrm{~kg}$ \\
Pelocity & $V$ & $947.6 \mathrm{~m} / \mathrm{s}$ \\
Dytch moment of inertia & $I_{y y}$ & $247.44 \mathrm{~kg} \cdot \mathrm{m}^{2}$ \\
Reference area & $Q$ & $293,638 \mathrm{~N} / \mathrm{m}^{2}$ \\
Reference diameter & $S$ & $0.04087 \mathrm{~m}^{2}$ \\
Gravitational acceleration & $g$ & $0.229 \mathrm{~m}$ \\
Time constant of tin actuator & $t_{1}$ & $9.8 \mathrm{~m} / \mathrm{s}^{2}$ \\
\hline
\end{tabular}

where $k_{q}$ is a chosen constant. The resultant control law is given by

$$
\begin{aligned}
u_{n d i c}= & \delta-\left[t_{1} /\left(b_{1}+k_{q} b_{2}\right)\right]\left\{k_{1}(y-\omega)\right. \\
& \left.+k_{2}\left[f_{1}+q+b_{1} \delta+k_{q}\left(f_{2}+b_{2} \delta\right)-\dot{\omega}\right]+m-\ddot{\omega}\right\},
\end{aligned}
$$

where

$$
\begin{gathered}
m=m_{1}\left[f_{1}(\alpha)+q+b_{1}(\alpha) \delta\right]+f_{2}(\alpha)+b_{2} \delta, \\
m_{1}=\frac{\partial f_{1}(\alpha)}{\partial \alpha}+\frac{\partial b_{1}(\alpha)}{\partial \alpha} \delta+k_{q} \frac{\partial f_{2}(\alpha)}{\partial \alpha}
\end{gathered}
$$

and $k_{1}, k_{2}$ are constant gains to be designed according to desired closed-loop behaviors.

Suppose that the command signal $\omega_{c m d}$ is filtered by a low-pass prefilter to provide the reference for tracking

$$
G(s)=\omega_{n}^{2} /\left(s^{2}+2 \varsigma \omega_{n} s+\omega_{n}^{2}\right)
$$

Substituting the NDIC law (33) into the longitudinal dynamics of the missile, the closed-loop error dynamics are given by

$$
\ddot{y}(t)-\ddot{\omega}(t)+k_{2}[\dot{y}(t)-\dot{\omega}(t)]+k_{1}[y(t)-\omega(t)]=0 .
$$

In this paper, the parameters in Eqs. (32)-(37) are chosen as

$$
\begin{gathered}
\varsigma=0.7, \omega_{n}=10(\mathrm{rad} / \mathrm{s}), \\
k_{q}=0.06(\mathrm{~s}), \\
k_{1}=15\left(1 / \mathrm{s}^{2}\right), k_{2}=6(1 / \mathrm{s}) .
\end{gathered}
$$

As shown in Eq. (37), the longitudinal dynamics of the missile is feedback linearized by NDIC. The closed-loop poles under NDIC is given by $-7.0 \pm 7.14 j$, thus promising tracking performance is achieved under the control law in the absence of disturbances. However, it is reported that such NDIC scheme has poor robustness and disturbance rejection ability [1, 22]. 


\subsection{NDOBRC for the missile system}

Define the state vector of the missile system as $\boldsymbol{x}=[\alpha, q, \delta]^{T}$. Rearranging the state equations of the missile systems (25), (26), (31) and the output equation (32), the formulation like affine nonlinear systems (1) is obtained. The concrete nonlinear vector-valued functions are denoted as

$$
\begin{gathered}
\boldsymbol{f}(\boldsymbol{x})=\left(\begin{array}{c}
f_{1}(\alpha)+q+b_{1}(\alpha) \delta \\
f_{2}(\alpha)+b_{2} \delta \\
-\left(1 / t_{1}\right) \delta
\end{array}\right), \\
\boldsymbol{g}_{1}(\boldsymbol{x})=\left(\begin{array}{c}
0 \\
0 \\
1 / t_{1}
\end{array}\right) \\
\boldsymbol{g}_{2}(\boldsymbol{x})=\left(\begin{array}{ccc}
1 & 0 & 0 \\
0 & 1 & 0 \\
0 & 0 & 1
\end{array}\right) \\
\boldsymbol{h}(\boldsymbol{x})=\alpha+k_{q} q .
\end{gathered}
$$

The lumped disturbance vector is

$$
\boldsymbol{d}=\left(\begin{array}{l}
d_{1} \\
d_{2} \\
d_{3}
\end{array}\right) .
$$

Using the nonlinear disturbance observer (2) introduced in Section 2, the lumped disturbances in the missile system can be estimated.

To determine the nonlinear disturbance compensation gain vector for the missile system following from the procedure proposed in Section 3, the above dynamics are reformulated as Eq. (17). The nonlinear matrix-value functions $\boldsymbol{g}_{1}(\boldsymbol{x})$ and $\boldsymbol{g}_{2}(\boldsymbol{x})$ are the same as those in Eqs. (42) and (43), while $\overline{\boldsymbol{f}}(\boldsymbol{x})$ and $\overline{\boldsymbol{h}}(\boldsymbol{x})$ are denoted as follows

$$
\begin{gathered}
\overline{\boldsymbol{f}}(\boldsymbol{x})=\left(\begin{array}{ccc}
f_{1}(\alpha) / \alpha & 1 & b_{1}(\alpha) \\
f_{2}(\alpha) / \alpha & 0 & b_{2} \\
0 & 0 & -1 / t_{1}
\end{array}\right), \\
\overline{\boldsymbol{h}}(\boldsymbol{x})=\left(\begin{array}{ccc}
1, & k_{q}, & 0
\end{array}\right) .
\end{gathered}
$$

Rearranging the NDIC law (33), gives

$$
u_{n d i c}=\overline{\boldsymbol{\alpha}}(\boldsymbol{x}) \boldsymbol{x}+\gamma(\omega, \dot{\omega}, \ddot{\omega}),
$$

where $\overline{\boldsymbol{\alpha}}(\boldsymbol{x})=\left[\bar{\alpha}_{1}(\boldsymbol{x}), \bar{\alpha}_{2}(\boldsymbol{x}), \bar{\alpha}_{3}(\boldsymbol{x})\right]$, and

$$
\bar{\alpha}_{1}(\boldsymbol{x})=-\frac{t_{1}}{b_{1}+k_{q} b_{2}}\left[k_{1}+\left(k_{2}+m_{1}\right) \frac{f_{1}(\alpha)}{\alpha}+\left(1+k_{2} k_{q}\right) \frac{f_{2}(\alpha)}{\alpha}\right],
$$




$$
\begin{gathered}
\bar{\alpha}_{2}(\boldsymbol{x})=-\frac{t_{1}}{b_{1}+k_{q} b_{2}}\left(k_{1} k_{q}+k_{2}+m_{1}\right), \\
\bar{\alpha}_{3}(\boldsymbol{x})=1-\frac{t_{1}}{b_{1}+k_{q} b_{2}}\left(k_{2} b_{1}+k_{2} k_{q} b_{2}+m_{1} b_{1}+b_{2}\right), \\
\gamma(\omega, \dot{\omega}, \ddot{\omega})=\frac{t_{1}}{b_{1}+k_{q} b_{2}}\left(k_{1} \omega+k_{2} \dot{\omega}+\ddot{\omega}\right) .
\end{gathered}
$$

Since all nonlinear functions including $\overline{\boldsymbol{f}}(\boldsymbol{x}), \overline{\boldsymbol{h}}(\boldsymbol{x}), \boldsymbol{g}_{\mathbf{1}}(\boldsymbol{x}), \boldsymbol{g}_{\mathbf{2}}(\boldsymbol{x})$ and $\overline{\boldsymbol{\alpha}}(\boldsymbol{x})$ have been obtained, the nonlinear disturbance compensation gain vector $\boldsymbol{\beta}(\boldsymbol{x})$ can be obtained by using Eq. (19).

The composite nonlinear disturbance observer based robust control (NDOBRC) law is given by

$$
u=u_{n d i c}+\boldsymbol{\beta}(\boldsymbol{x}) \hat{\boldsymbol{d}}
$$

where $u_{n d i c}$ is the NDIC law (33), $\boldsymbol{\beta}(\boldsymbol{x})$ is the disturbance compensation vector given by (19), and $\hat{\boldsymbol{d}}$ is disturbance estimate governed by (2). The control structure of the nonlinear disturbance observer based NDIC scheme is shown in Fig. 1.

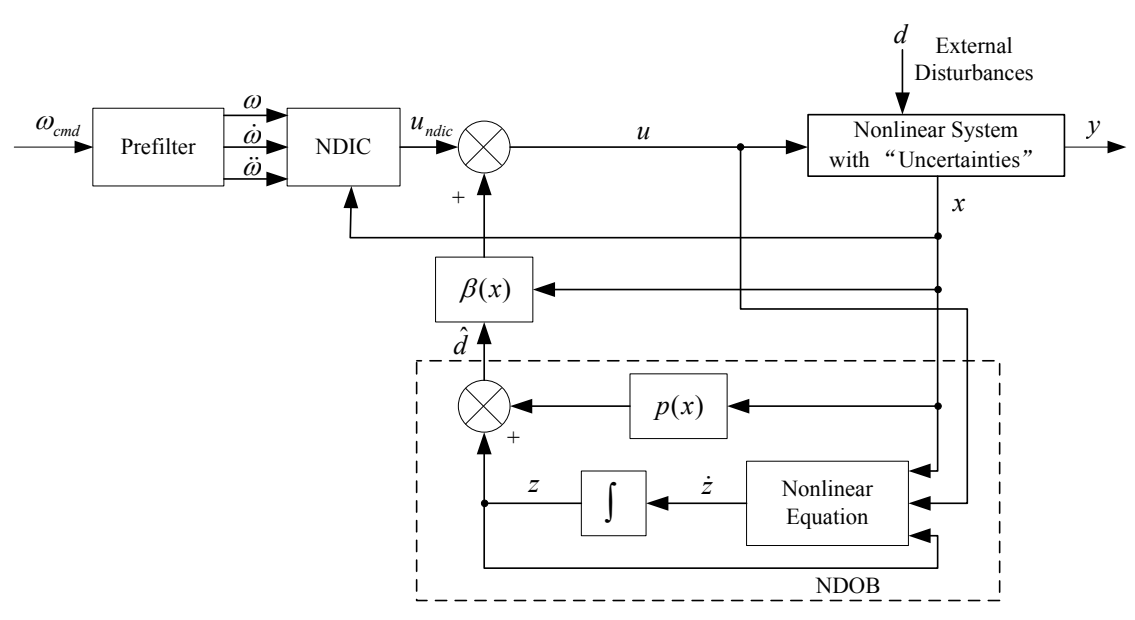

Figure 1: Control structure of nonlinear disturbance observer based robust control (NDOBRC) scheme.

\section{Simulation studies}

In this paper, the nonlinear vector-valued function $\boldsymbol{l}(\boldsymbol{x})$ in NDOB (2) is chosen as

$$
\boldsymbol{l}(\boldsymbol{x})=\left(\begin{array}{ccc}
10 & 0 & 0 \\
0 & 10 & 0 \\
0 & 0 & 10
\end{array}\right)
$$

It is reported that the NDIC method has a poor disturbance rejection ability and robustness [1]. Integral action is a practical and most widely used method to eliminate the steady-state error in the presence of disturbances/uncertainties [17]. To demonstrate the effectiveness of the proposed method, in addition to the NDIC method, NDIC plus an integral action (called NDIC+I) is also 
employed for comparison. The control law of the NDIC+I is represented as follows

$$
\begin{aligned}
& u_{n d i c+i}=\delta-\left[t_{1} /\left(b_{1}+k_{q} b_{2}\right)\right]\left\{k_{0} \int(y-\omega) d \tau+k_{1}(y-\omega)\right. \\
& \left.+k_{2}\left[f_{1}+q+b_{1} \delta+k_{q}\left(f_{2}+b_{2} \delta\right)-\dot{\omega}\right]+m-\ddot{\omega}\right\},
\end{aligned}
$$

where the integral coefficient is chosen as $k_{0}=20$ to achieve a satisfactory performance.

It can be seen from the control law (55) that the NDIC+I method has a PID like structure. Furthermore, the closed-loop system under Eq. (55) is given by

$$
\ddot{y}-\ddot{\omega}+k_{2}(\dot{y}-\dot{\omega})+k_{1}(y-\omega)+k_{0} \int(y-\omega) d \tau=0 .
$$

As will be shown by simulation later, the offset caused by disturbances and uncertainties can be eliminated by the NDIC+I method.

\subsection{External disturbance rejection ability}

In this subsection, the external disturbance rejection ability of the missile system under the proposed NDOBRC method is investigated. Considering the case that the external disturbances $d_{1}=1$ is imposed on systems at $t=6 \mathrm{sec}$ while $d_{2}=5$ enters at $t=2 \mathrm{sec}$, the response curves of both the output and input under three control methods are shown in Fig. 2. The corresponding response curves of the states are shown in Fig. 3.

It can be observed from Fig. 2(a) that the NDIC method results in a large steady-state error. As for the NDIC+I method, there is no steady-state error any more, but large overshoot and long settling time are experienced. The proposed NDOBRC exhibits a much better transient and steady-state performance, such as small overshoot, short settling time and zero steady-state error. The control profile in Fig. 2(b) shows that no excessive (or) high gain is demanded for all the three methods.

Fig. 3 shows that all states under the three methods remain within allowable regions. This means that the proposed method gains much better external disturbance rejection performance than the other two methods without bringing adverse effects to all the states.

\subsection{Robustness against model uncertainties}

The robustness against model uncertainties of the proposed NDOBRC is tested in this part. To investigate the performance of robustness in detail, three cases of model uncertainties are considered.

Case I: both $f_{1}(\alpha)$ and $f_{2}(\alpha)$ have variations of $+20 \%$.

For the first case of model uncertainties, the response curves of the output/input and the states under the three control methods are shown in Figs. 4 and 5, respectively. It can be observed from Fig. 4(a) that the NDIC scheme has resulted in large steady-state error. For the NDIC+I method, the steady-state error was eliminated but quite slowly. The proposed NDOBRC method approaches to the reference setpoint rapidly without steady-state error.

As shown in Figs. 4(b) and 5, both the control input and the states remain within allowable 


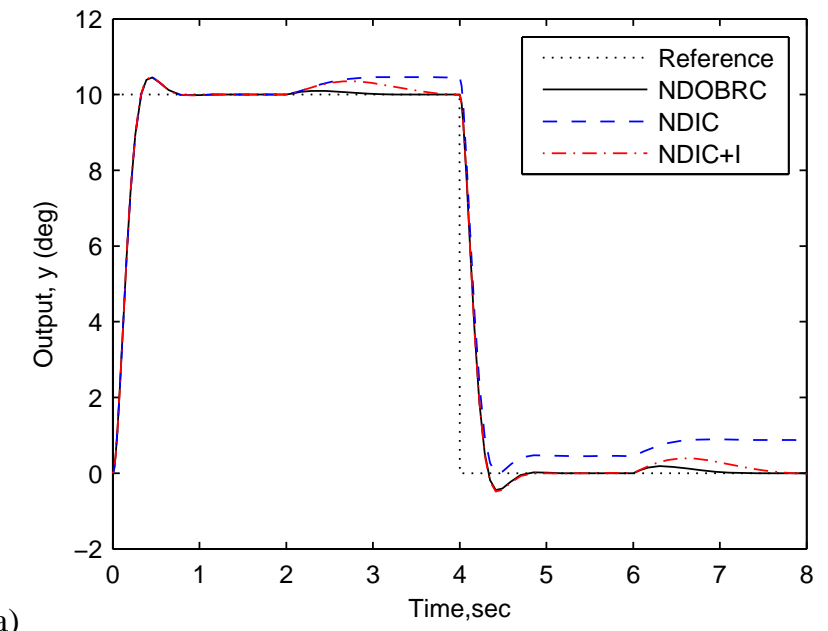

(a)

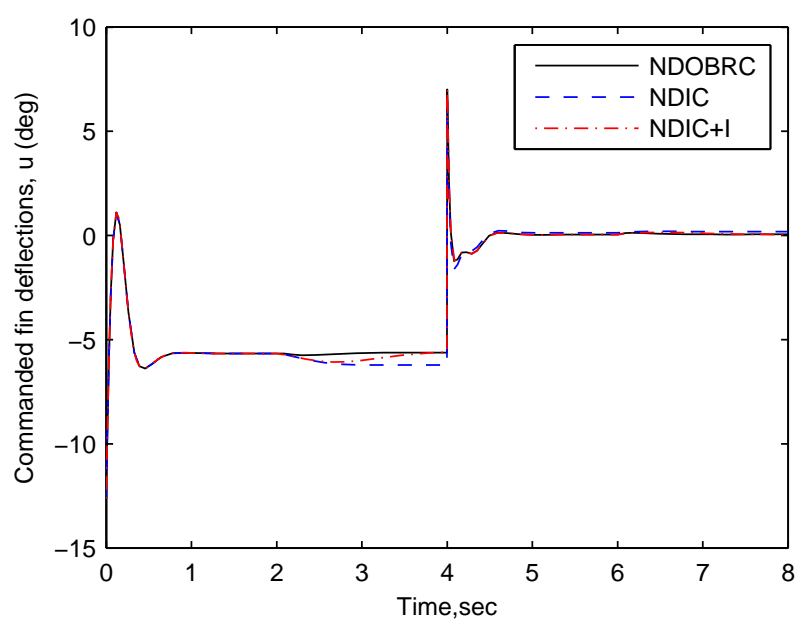

Figure 2: Response curves of the output and input in the presence of external disturbances under the control laws of NDOBRC (53) (solid line), NDIC (33) (dashed line), and NDIC+I (55) (dash-dotted line). The reference signal is denoted by dotted line.

regions. These variables under the NDOBRC method converge to the desired equilibrium point much quicker than those under the other two methods.

Case II: $f_{1}(\alpha)$ and $f_{2}(\alpha)$ have variations of $-20 \%$ and $-5 \%$, respectively.

In such case, Figs. 6 and 7 show the response curves of the output/input and states under the three control methods. It can be observed from Fig. 6(a) that the output under the NDIC method substantially departs from the desired reference trajectory. Essentially, this implies that the missile becomes unstable as the model is only valid when $|\alpha| \leq 20$ degrees, but the angle of attack reaches 70 degrees in simulation. Fig. 6(a) shows that the output under the NDIC+I method asymptotically approaches to the setpoint with oscillation and large overshoot. The proposed NDOBRC has achieved the best performance, including a small overshoot, a short settling time, no oscillation and zero steady-state error.

As shown in Fig. 6(b), the magnitude of the control input under the NDOBRC is much smaller 
than those under the other two methods. The NDIC method is unusable in this case because the magnitude of the control input is huge and over the actuator constraint. Also from the response curves of the states in Fig. 7, it can be concluded that the proposed method gains the best performance of robustness among all the three methods.

Case III: $f_{1}(\alpha)$ and $f_{2}(\alpha)$ have variations of $-20 \%$ and $-7 \%$, respectively.

The case of an even more severe model uncertainties is investigated in this part. The response curves of the output/input and states are shown in Figs. 8 and 9, respectively.

In this case, the control performances under the NDOBRC and NDIC are quite similar with those in Case II. However, the response curves under the NDIC+I method becomes oscillating and unstable. This shows that the proposed NDOBRC achieves much better robust performance and stability compared with other two methods.

\section{Conclusion}

As clearly demonstrated in the missile example in this paper, external disturbances, unmodeled dynamics and parameter perturbations always bring adverse effects to stability and performance of control systems. Disturbance observer based control (DOBC) has provided a solution to this problem. It can significantly improve disturbance attenuation ability and robustness against uncertainties, and acts like a "patch" to the existing design without considerably changing the nominal control design. Existing DOBC methods were only applicable to matched disturbances. To this end, a nonlinear disturbance observer based robust control (NDOBRC) method has been proposed for nonlinear systems in the presence of mismatched disturbances and uncertainties. It is shown that by appropriately design the nonlinear compensation gains, zero steady-state tracking error can be achieved on system output. Simulation studies of a missile system have been carried out to demonstrate the validity of the proposed NDOBRC method. The results have shown that the proposed method obtains much better disturbance rejection ability and robustness against model uncertainties as compared with nonlinear dynamic inversion control (NDIC) and nonlinear dynamic inversion control plus integral action (NDIC+I) methods.

\section{Acknowledgements}

This work was supported in part by Natural Science Foundation of Jiangsu Province (BK2008295), Science Foundation of Aeronautics (20080769006), Graduate Innovation Program of Jiangsu Province (CX10B_077Z), and Scientific Research Foundation of Graduate School of Southeast University. J. Yang would like to thank the China Scholarship Council (CSC) for supporting his visiting in the UK. He also acknowledges the Department of Aeronautical and Automotive Engineering for hosting his research at Loughborough University. 


\section{References}

[1] Chen, W.-H.: 'Nonlinear disturbance observer-enhanced dynamic inversion control of missiles', J. Guid. Control Dyn., 2003, 26, (1), pp. 161-166

[2] Yao, B., Majed, M.A., and Tomizuka, M.: 'High-performance robust motion control of machine tools: an adaptive robust control approach and comparative experiments', IEEE/ASME Trans. Mechatron., 1997, 2, (2), pp. 63-76

[3] Chen, W.-H.: 'Disturbance observer based control for nonlinear systems', IEEE/ASME Trans. Mechatron., 2004, 9, (4), pp. 706-710

[4] Ohishi, K., Nakao, M., Ohnishi, K., and Miyachi, K.: 'Microprocessor-controlled DC motor for load-insensive position servo system', IEEE Trans. Ind. Electron., 1987, 34, (1), pp. 44-49

[5] Komada, S., Machii, N., and Hori, T.: 'Control of redundant manipulators considering order of disturbance observer’, IEEE Trans. Ind. Electron., 2000, 47, (2), pp. 413-420

[6] Ishikawa, J., and Tomizuka, M.: 'A novel add-on compensator for cancellation of pivot nonlinearities in hard disk drives', IEEE Trans. Magn., 1998, 34, (4), pp. 1895-1897

[7] Kempf, C.J., and Kobayashi, S.: 'Disturbance observer and feedforward design for a highspeed direct-drive position table', IEEE Trans. Control Syst. Technol., 1999, 7, (5), pp. 513-526

[8] Chen, X.S., Yang, J., Li, S.H., and Li, Q.: 'Disturbance observer based multi-variable control of ball mill grinding circuits', J. Process Control, 2009, 19, (7), pp. 1205-1213

[9] Yang, J., Li, S.H., Chen, X.S., and Li, Q.: 'Disturbance rejection of ball mill grinding circuits using DOB and MPC', Powder Technol., 2010, 198, (2), pp. 219-228

[10] Yang, Z.-J., Tsubakihara, H., Kanae, S., Wada, K., and Su, C.-Y.: 'A novel robust nonlinear motion controller with disturbance observer', IEEE Trans. Control Syst. Technol., 2008, 16, (1), pp. 137-147

[11] Chen, W.-H., Ballance, D.J., Gawthrop, P.J., and O'Reilly, J.: 'A nonlinear disturbance observer for robotic manipulators', IEEE Trans. Ind. Electron., 2000, 47, (4), pp. 932-938

[12] Guo, L., and Chen, W.-H.: 'Disturbance attenuation and rejection for systems with nonlinearity via DOBC approach', Int. J. Robust Nonlinear Control, 2005, 15, (3), pp. 109-125

[13] Wei, X.J., and Guo, L.: 'Composite disturbance-observer-based control and terminal sliding mode control for non-linear systems with disturbances', Int. J. Control, 2009, 82, (6), pp. 1082-1098

[14] Chen, X.K., Komada, S., and Fukuda, T.: 'Design of a nonlinear disturbance observer', IEEE Trans. Ind. Electron., 2000, 47, (2), pp. 429-436

[15] Wei, X.J., and Guo, L.: 'Composite disturbance-observer-based control and H-infinity control for complex continuous models', Int. J. Robust Nonlinear Control, 2010, 20, (1), pp. 106-118

[16] Barmish, B., and Leitmann, G.: 'On ultimate boundedness control of uncertain systems in the absence of matching assumptions', IEEE Trans. Autom. Control, 1982, 27, (1), pp. 153-158

[17] Lin, C.-F., Cloutier, J.R., and Evers, J.H.: 'High-performance, robust, bank-to-turn missile autopilot design', J. Guid. Control Dyn., 1995, 18, (1), pp. 46-53

[18] Chen, W.-H., Ballance, D.J., Gribble, J.J., and O'Reilly, J.: 'Nonlinear PID predictive controller', IEE Proc.-Control Theory Appl., 1999, 146, (6), pp. 603-611

[19] Isidori, A.: 'Nonlinear Control Systems II' (Springer-Verlag, London, 1999)

[20] Lu, P.: 'Nonlinear predictive controllers for continuous systems', J. Guid. Control Dyn., 1994, 17, (3), pp. 553-560

[21] Lane, S.H., and Stengel, R.F.: 'Flight control design using non-linear inverse dynamics," $A u$ tomatica, 1988, 24, (4), pp. 471-483

[22] Schumacher, C., and Khargonekar, P.P.: 'Missile autopilot designs using $\mathrm{H}_{\infty}$ control with gain scheduling and dynamic inversion', J. Guid. Control Dyn., 1998, 21, (2), pp. 234-243 


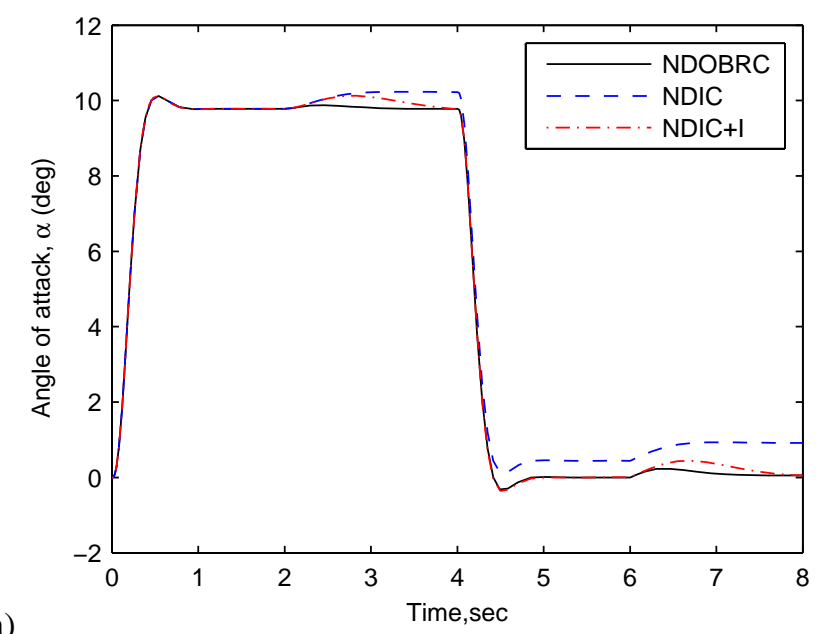

(a)

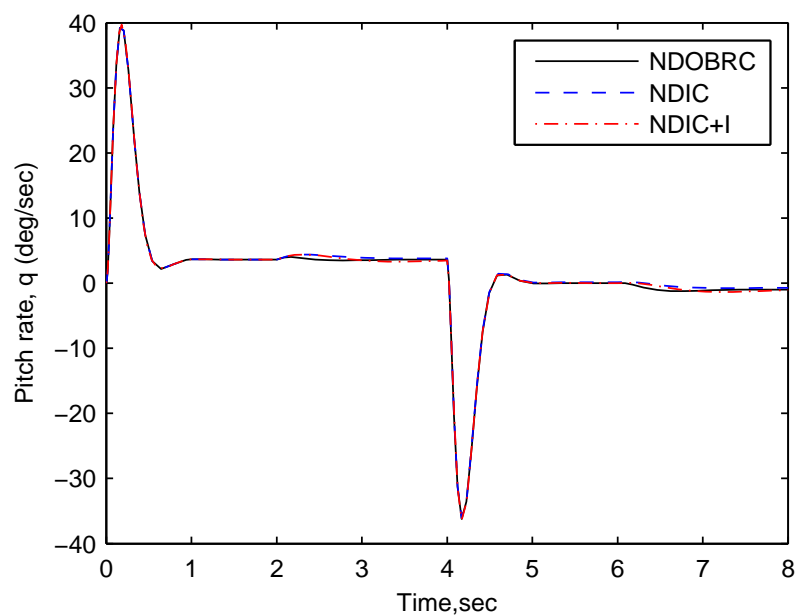

(b)

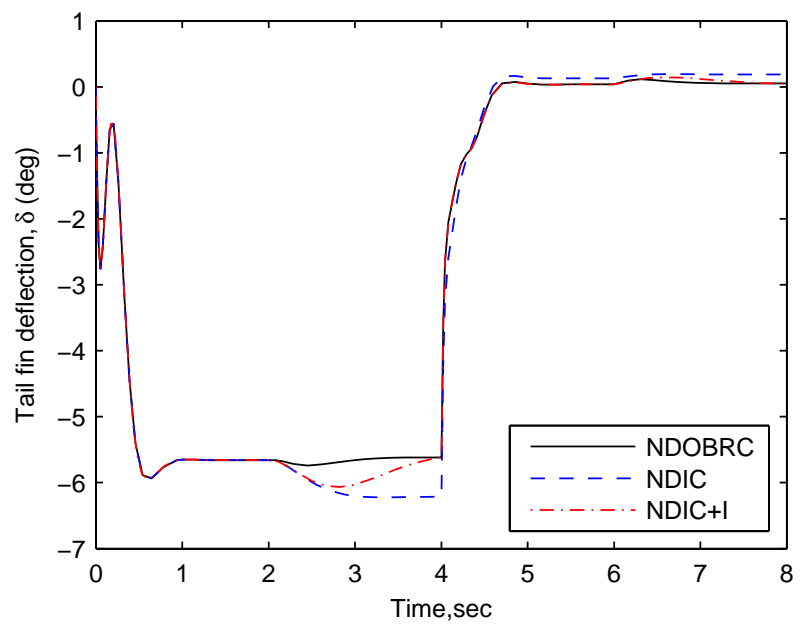

Figure 3: Response curves of the states in the presence of external disturbances under the control laws of NDOBRC (53) (solid line), NDIC (33) (dashed line), and NDIC+I (55) (dash-dotted line). 


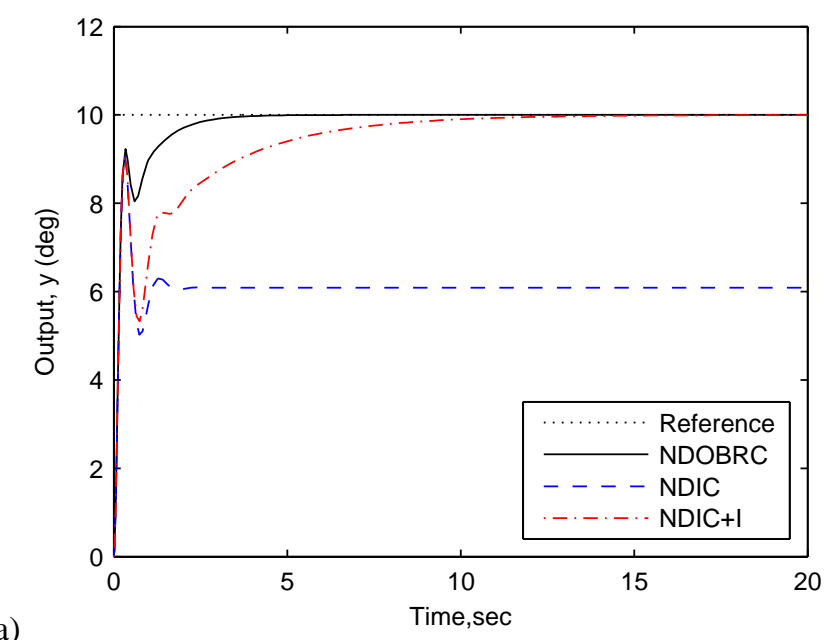

(a)

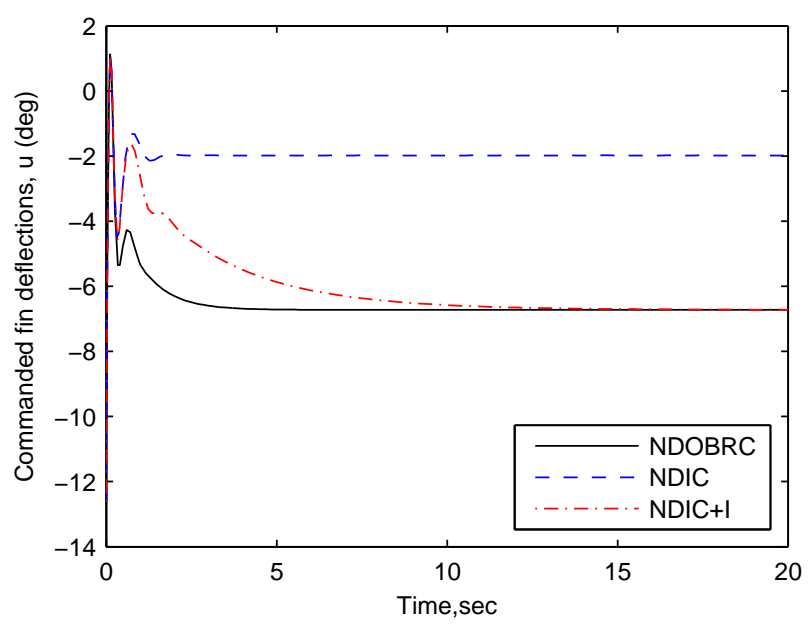

Figure 4: Response curves of the output and input in the first case of model uncertainties (both $f_{1}(\alpha)$ and $f_{2}(\alpha)$ have variations of $\left.+20 \%\right)$ under the control laws of NDOBRC (53) (solid line), NDIC (33) (dashed line), and NDIC+I (55) (dash-dotted line). The reference signal is denoted by dotted line. 


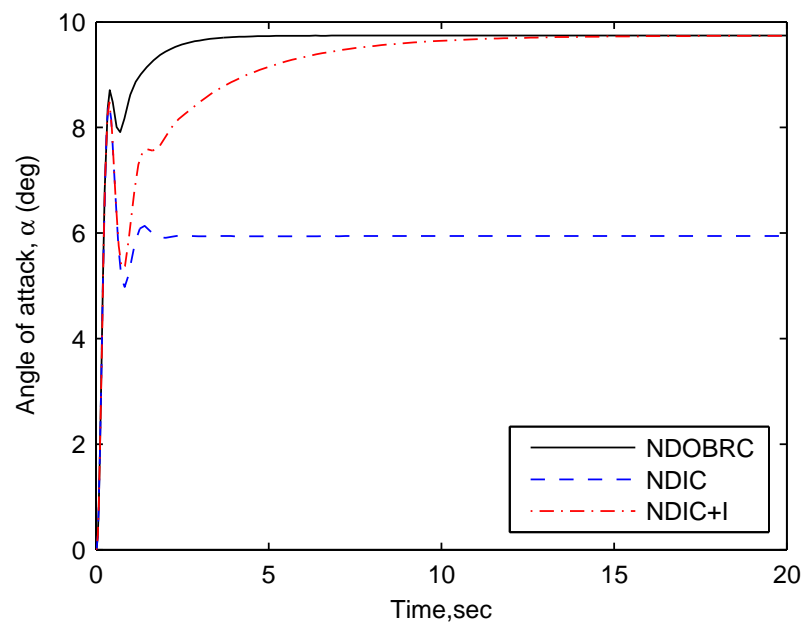

(a)

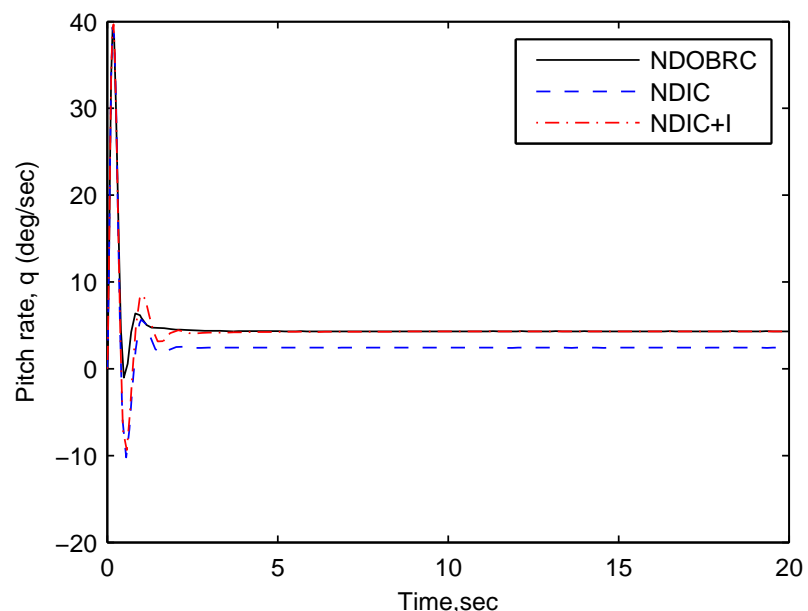

(b)

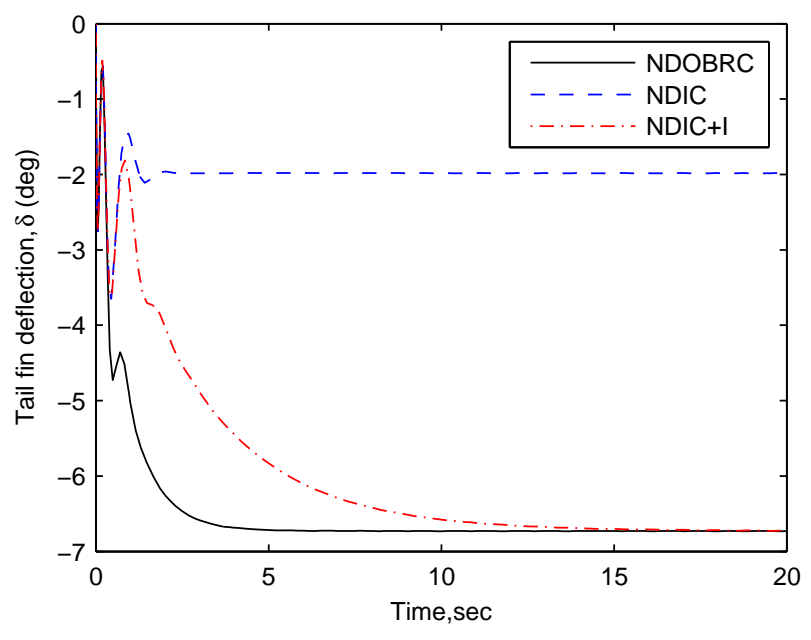

(c)

Figure 5: Response curves of the states in the first case of model uncertainties (both $f_{1}(\alpha)$ and $f_{2}(\alpha)$ have variations of $+20 \%$ ) under the control laws of NDOBRC (53) (solid line), NDIC (33) (dashed line), and NDIC+I (55) (dash-dotted line). 


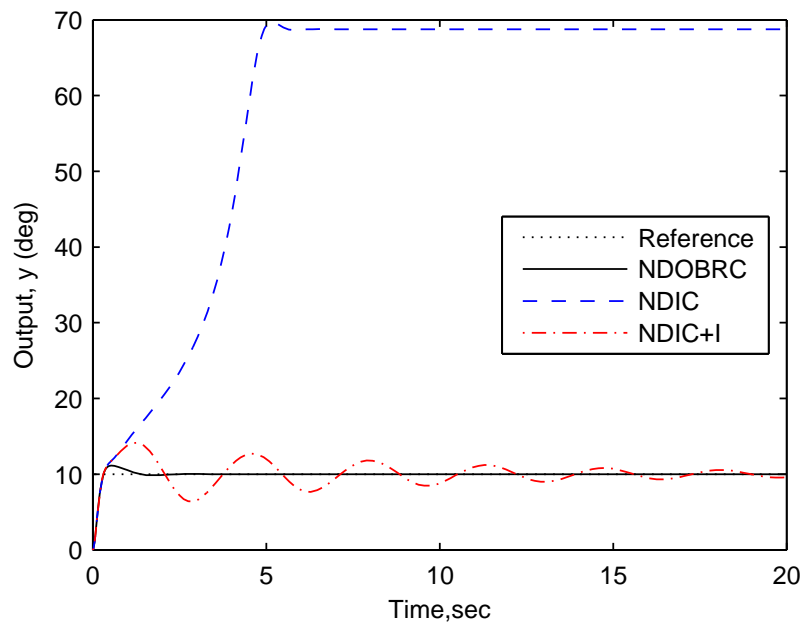

(a)

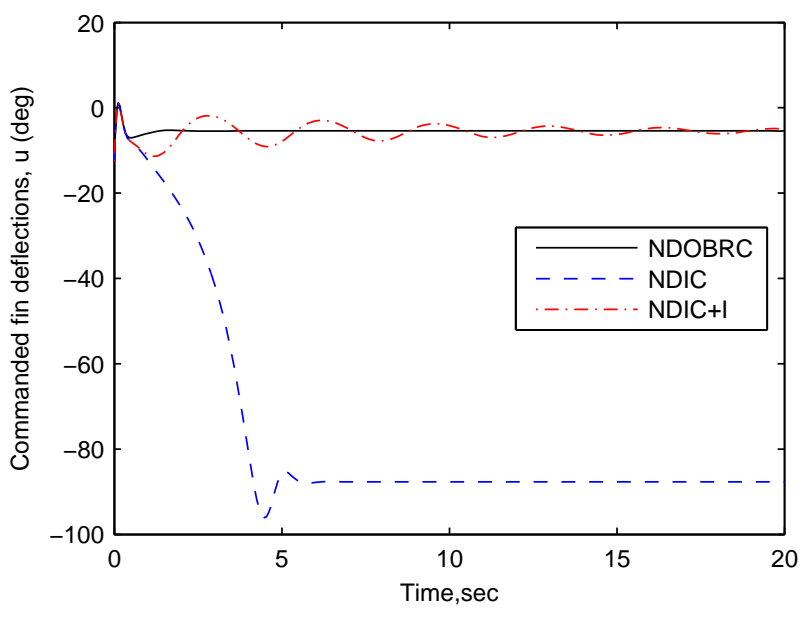

Figure 6: Response curves of the output and input in the second case of model uncertainties $\left(f_{1}(\alpha)\right.$ and $f_{2}(\alpha)$ have variations of $-20 \%$ and $-5 \%$, respectively) under the control laws of NDOBRC (53) (solid line), NDIC (33) (dashed line), and NDIC+I (55) (dash-dotted line). The reference signal is denoted by dotted line. 


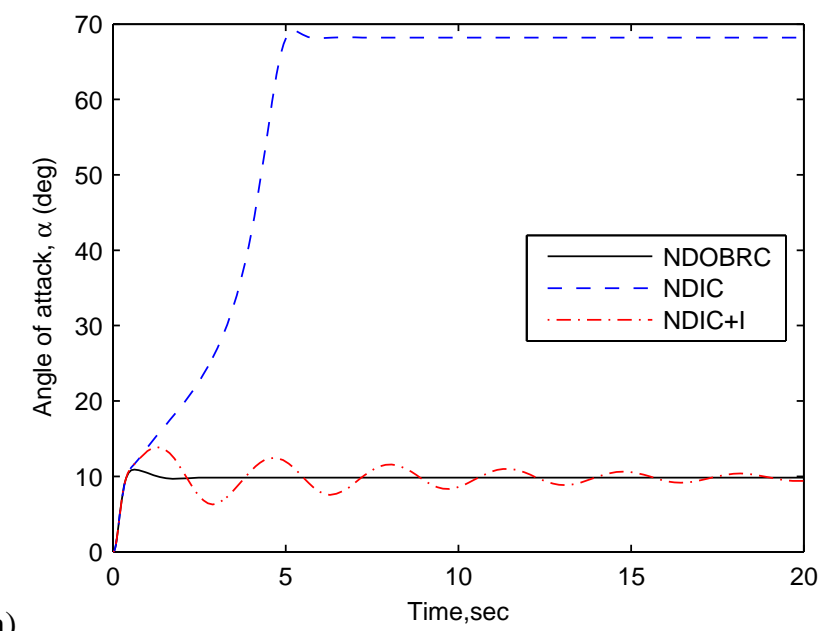

(a)

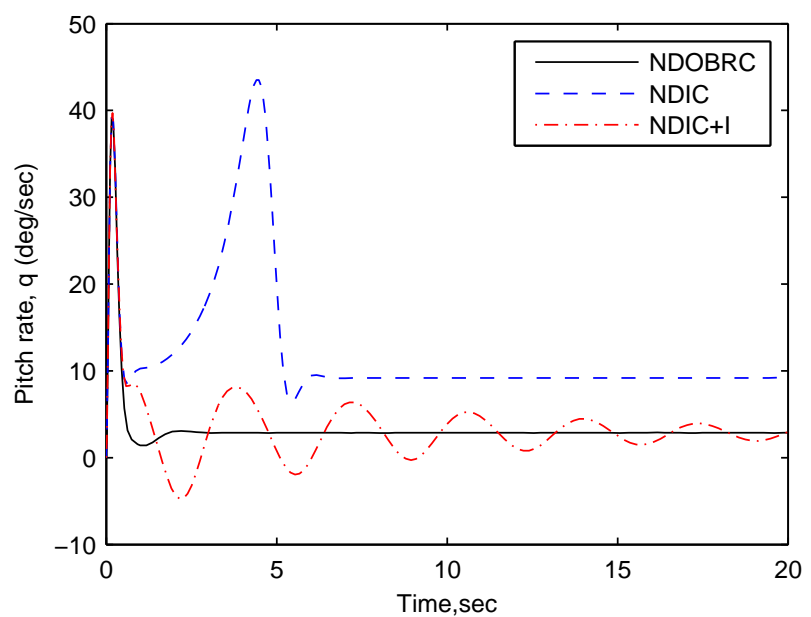

(b)

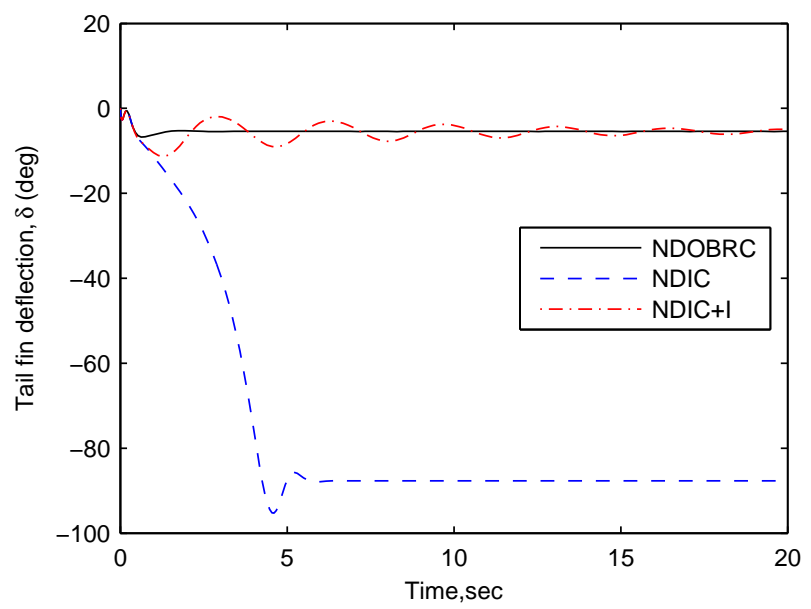

Figure 7: Response curves of the states in the second case of model uncertainties $\left(f_{1}(\alpha)\right.$ and $f_{2}(\alpha)$ have variations of $-20 \%$ and $-5 \%$, respectively) under the control laws of NDOBRC (53) (solid line), NDIC (33) (dashed line), and NDIC+I (55) (dash-dotted line). 


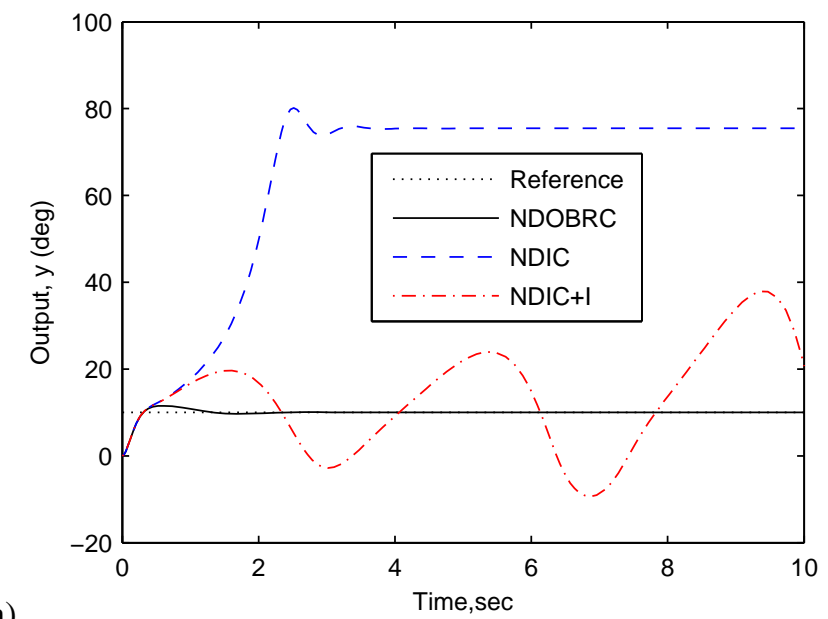

(a)

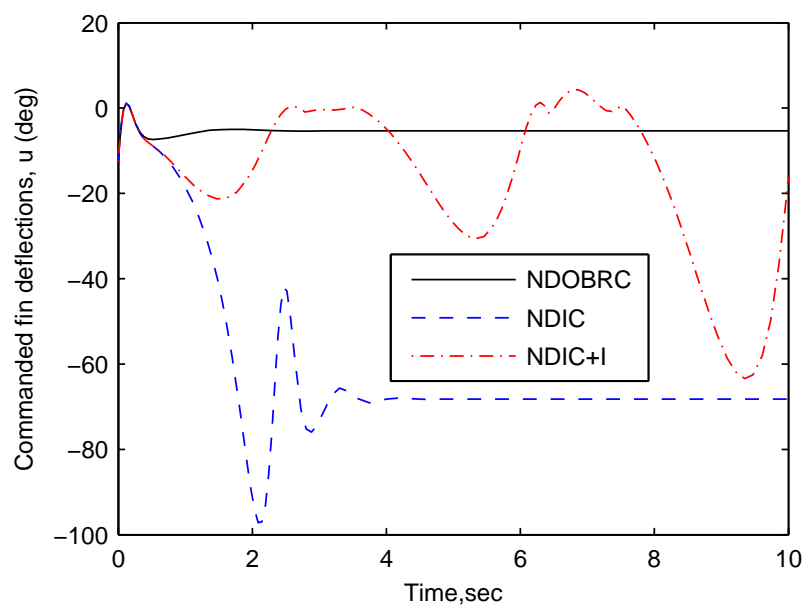

Figure 8: Response curves of the output and input in the third case of model uncertainties $\left(f_{1}(\alpha)\right.$ and $f_{2}(\alpha)$ have variations of $-20 \%$ and $-7 \%$, respectively) under the control laws of NDOBRC (53) (solid line), NDIC (33) (dashed line), and NDIC+I (55) (dash-dotted line). The reference signal is denoted by dotted line. 


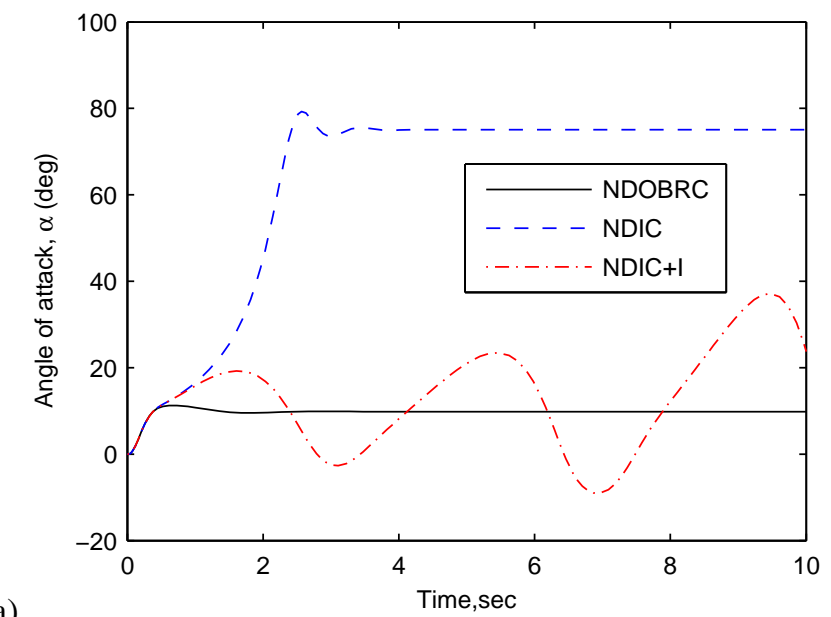

(a)

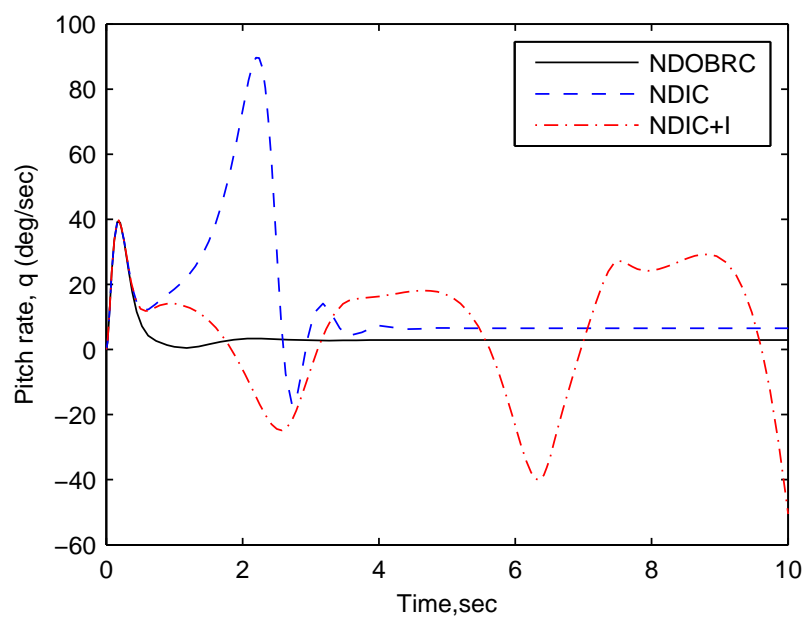

(b)

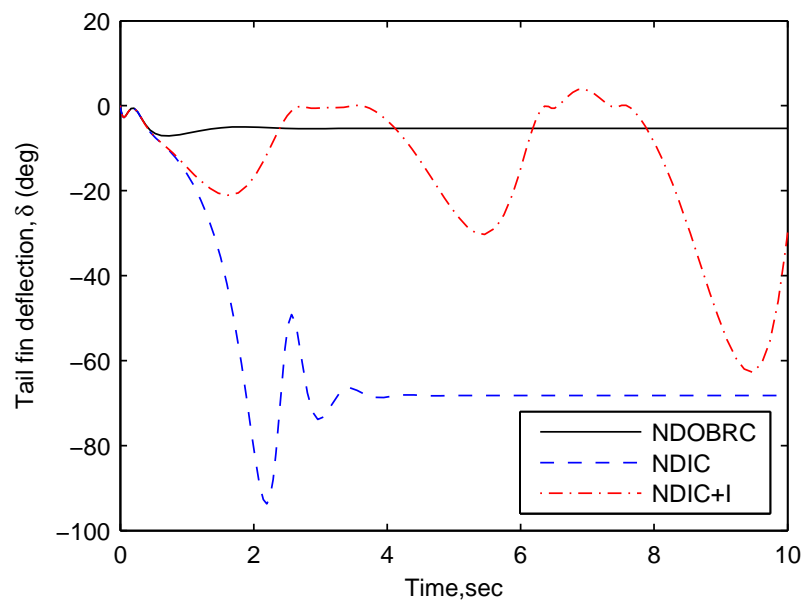

Figure 9: Response curves of the states in the third case of model uncertainties $\left(f_{1}(\alpha)\right.$ and $f_{2}(\alpha)$ have variations of $-20 \%$ and $-7 \%$, respectively) under the control laws of NDOBRC (53) (solid line), NDIC (33) (dashed line), and NDIC+I (55) (dash-dotted line). 